Check for updates

Cite this: DOI: 10.1039/d1me90037d

\section{Introduction to nature-inspired solutions for engineering}

\author{
Marc-Olivier Coppens (iD *a and Bharat Bhushan (iD ${ }^{b}$
}

DOI: 10.1039/d1me90037d

rsc.li/molecular-engineering

Nature holds a treasure trove of ideas to solve challenging engineering problems. Biological systems had to evolve to address multiple objectives under constraints. Biological materials and systems typically need to satisfy many

\footnotetext{
${ }^{a}$ Centre for Nature-Inspired Engineering (CNIE), Department of Chemical Engineering, University College London (UCL), Torrington Place, London, WC1E 7JE, UK. E-mail: m.coppens@ucl.ac.uk ${ }^{b}$ Nanoprobe Laboratory for Bio- \& Nanotechnology and Biomimetics (NLBB), The Ohio State University, 201 W. 19th Avenue, Columbus, Ohio, 43210-1142, USA.E-mail: bhushan.2@osu.edu
}

functions simultaneously, such as scalability, robustness, and various mechanical properties under static and dynamic conditions. In addition, the efficient use of materials and energy resources could be an existential question for an organism to survive. Similar goals exist for many materials and systems engineering problems, and increasingly so, because of pressing sustainable development goals. Hence, it seems "natural" to look at nature for inspiration.

Nevertheless, fundamental mechanistic understanding is crucial to avoid the trap of narrow biomimicry. Copying nature will rarely achieve the desired outcomes, as the context of technological problems often differs from that in biology, such as the available building blocks, practical synthesis and manufacturing methods, or environmental conditions, not to forget the socio-economic requirements of technology. Hence, nature-inspired solutions for engineering require grounding in fundamentals, and a systematic design methodology that is cognisant of the applied context.

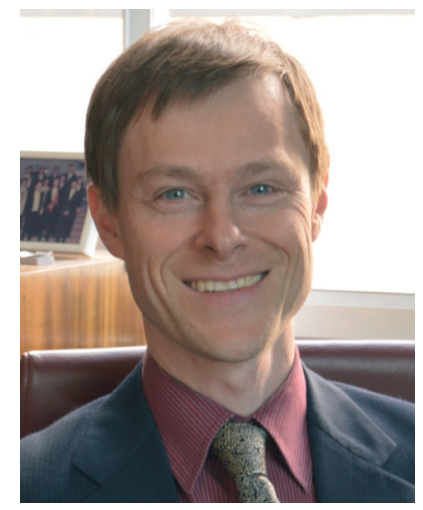

Marc-Olivier Coppens
Marc-Olivier Coppens has been the Ramsay Memorial Professor in Chemical Engineering at UCL since 2012, after professorships at Rensselaer and TU Delft. He is the Vice-Dean of Engineering (Interdisciplinarity, Innovation) and directs the Centre for Nature-Inspired Engineering, which was granted EPSRC "Frontier Engineering" (2013) and "Progression" (2019) Awards. He is recognised for pioneering nature-inspired chemical engineering (NICE) and a systematic nature-inspired solution methodology, where the fundamental mechanisms underpinning desirable traits in nature are leveraged to accelerate innovation. He is Fellow of the RSC, AIChE and IChemE, corresponding member of the Saxon Academy of Sciences (Germany), and Qiushi Professor at Zhejiang University.

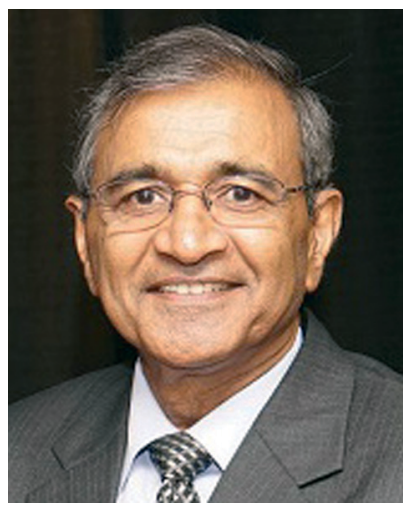

Bharat Bhushan
Dr. Bharat Bhushan is an Academy Professor (San Jose, CA), and has served as an Ohio Eminent Scholar, Howard D. Winbigler Professor, and Director of the Nanoprobe Laboratory for Bio- \& Nanotechnology and Biomimetics at Ohio State University (Columbus, OH). $\mathrm{He}$ holds two M.S., a Ph.D. in mechanical engineering, an MBA, and five honorary doctorates. His research interests include fundamental studies in the interdisciplinary areas of bio/nanotribology/nanomechanics, nanomaterials characterization, scanning probe techniques, magnetic storage, bio/nanotechnology, nanomanufacturing, bioinspired liquid repellency, self-cleaning, anti-icing, anti-fouling, water harvesting and science and technology policy. He has authored 10 books and 900+ papers and holds 25 US and foreign patents. He has previously worked for Mechanical Technology Inc., SKF, and the IBM Almaden Research Center, San Jose, CA. 
This themed collection brings together 8 articles that discuss widely different topics but are united in their vision to gain insights from fundamentals in natural, molecular systems for the design and engineering of novel functional materials and devices. Some of the articles present original research, while other ones review recent work, and two present a fresh methodological perspective. The idea of this themed collection arose through the 1st ECI Conference on Nature-Inspired Engineering, held in Cetraro, Italy, in 2019, organised by the Guest Editors, and where several of the authors met and presented.

In this issue, Goldfield and Coppens (DOI: 10.1039/D0ME00062K) introduce a systematic nature-inspired solution (NIS) methodology to accelerate developmental bioengineering, a new form of therapy that recapitulates biological development for repair. The paper merges the thinking that underpins the biomedical research into bio-inspired engineering at the Harvard Wyss Institute and into nature-inspired chemical engineering (NICE) and NIS at the UCL Centre for Nature-Inspired Engineering (CNIE). It discusses the potential of a NIS methodological framework through two recent medical breakthroughs that present tremendous hope to patients: retinal pigment epithelium repair for age-related macular degeneration, which leads to blindness, and recovery from paralysisprovoking spinal cord injuries.

Certain animals and plants in the desert can collect water from fog without energy input. How they achieve this could inspire solutions for accessing fresh water in coastal deserts. Zheng and co-workers (DOI: 10.1039/ D1ME00019E) discuss recent advances in such biomimetic fog harvesting systems, focusing on improving their efficiency and large-scale fabrication. They learn from the multiscale structural features of desert beetles and cactus thorns, and how droplets interact with these structures, to develop fabrics and devices that emulate natureinspired geometrical and physicochemical properties to endow them with similar fast water collection, transport, and removal.

In their review on amine-assisted silica synthesis methods, Manning et al. (DOI: 10.1039/D0ME00131G) note that the difficulty faced in replicating the complexity of naturally occurring biosilica materials is partly due to a lack of "joined-up", methodological research. They present a unified mechanistic interpretation of formation that advances the understanding of the structure-function relationships between amine additives and the ultimate materials. This should enable the design of more complex functional materials, based on experiments and computations, from the molecular to the production scale.

Gurera and Bhushan (DOI: 10.1039/ D0ME00099J) show how bioinspired conical and triangular surfaces can be used to move gas bubbles, even against gravity or buoyancy. This matters in applications ranging from biomedicine and drag reduction for ships, to aeration in food production, water treatment and surface cleaning. Like Zheng et al., they draw inspiration from cacti and spider webs, although for gas bubbles rather than water droplets; the fundamental mechanism behind the bubble movement is the Laplacian pressure gradient inside the bubbles.

Korley and co-workers (DOI: 10.1039/ D1ME00043H) show how peptidepolyurea hybrid materials can be engineered to create hierarchical structures and thermo-responsive shape memory, influenced by the peptide secondary structure (the ratio of $\alpha$-helices and $\beta$-sheets), through interand intra-molecular hydrogen bonding arrangements and microphaseseparated morphology. The authors are inspired by spider silk and other natural materials, where the tunable properties are driven by a similar modulation of the structural organisation. Smart, stimuli-responsive, functional materials could be designed using these principles by appropriate bio-inspired force balancing and structural organisation.

Stachewicz (DOI: 10.1039/ D1ME00066G) discusses how lessons could be learnt from microscopy studies on polar bear hair to design tougher materials. Polar bear hair has exceptional mechanical properties, thanks to energy dissipation and crack deflection, in combination with superior thermal insulation. These multifunctional properties are induced by the hair's multiscale structure.

Malyuk and Ivanova (DOI: 10.1039/ D0ME00052C) create adaptive liquid lenses that are actuated by a laser beam to modulate the thermocapillary and solutocapillary forces, which can be converging or diverging. The eye's liquid lens serves as a source of inspiration, due to its robustness and the extreme sensitivity of the interfacial curvature and the focal length to external stimuli. The authors create bio-inspired lenses containing ethylene glycol, ethanol, or benzyl alcohol. Their paper discusses two effects that could impact the stability and ability to switch between different focal lengths, namely tilting and evaporation due to heating. This is important for the practical, repeated and stable use of the lenses in various optical devices.

Fouling is a major issue hampering industrial membrane separations used in water treatment and protein separations. Mohamed et al. (DOI: $10.1039 /$ D0ME00045K) show how the fouling of polyester membranes could be reduced by learning from the remarkable anti-fouling properties of the glomerular complex in kidneys. Here, hydrophilicity and negatively charged polysaccharide chains lead to, respectively, a hydration layer and electrostatic repulsion of foulants. By employing a systematic approach using different grafted polymers on polyester membranes, the authors demonstrate that both hydrophilicity and charge play a role, with charge having the largest impact.

We hope that the breadth of subjects in these articles may motivate readers to explore the vast opportunities of natureinspired engineering to accelerate discovery and innovation in molecular systems design and engineering - by following a mechanistic, methodological approach, rather than sheer imitation of nature out of context. 